\title{
Prenatal Diagnosis of Uhl Anomaly with Autopsy Correlation
}

\author{
Saji Philip, $\mathrm{PhD}^{1}$ \\ Sarasa Bharati, $\mathrm{PhD}^{2}$ \\ ${ }^{1}$ Fetal Cardiac Services, Division of Pediatric Cardiolo \\ Cardio-Vascular Centre, Parumala, Kerala, India \\ ${ }^{2}$ Division of Cardiac Pathology, Frontier Mediville, Frontier Life Line \\ Hospital, Chennai, India \\ ${ }^{3}$ Dr. K M. Cherian Heart Foundation, Frontier Life Line hospital, Chennai, India \\ ${ }^{4}$ Department of Pathology, Rush University Medical Center, Chicago, \\ University of Illinois at Chicago, Rosalind Franklin University of \\ Sciences, North Chicago, Illinois \\ ${ }^{5}$ The Advocate Children's Hospital, Oak Lawn, Illinois
}

Am J Perinatol Rep 2016;6:e91-e95.
Address for correspondence Dr. Saji Philip, PhD, FAHA, St. Gregorios Cardio-Vascular Centre, Dr. K M. Cherian Heart Foundation, Parumala, Pathanamthitta District, Kerala, India 689626

(e-mail: tfcsaji@yahoo.co.in; tfcsaji@hotmail.com).

Abstract
Keywords
- dilated right ventricle
- fetal autopsy
- right ventricular
dysplasia
- Uhl anomaly

Uhl anomaly is a rare form of congenital hypoplasia of the right ventricular myocardium. Here, we report, a rare finding in fetal cardiac ultrasound in a 33-year-old woman who presented at 20 weeks' of gestation. A diagnosis of Uhl anomaly was made. An autopsy was performed at 23 weeks gestation after obtaining permission for medicolegal termination of pregnancy. Histopathological examination confirmed the diagnosis. Diagnosing Uhl anomaly in fetal life is essential since mortality and survival mainly depend on the severity of right ventricle dysfunction related to, the either partial or complete absence of the myocardium. Hence, surviving cases need to be followed up carefully and counselled accordingly.
Uhl anomaly is a rare form of congenital hypoplasia of the right ventricular myocardium. It could be partial or there could be a total absence of the myocardium of the right ventricle (RV) with normal tricuspid valve, intact ventricular septum, and left ventricular myocardium. ${ }^{1-4}$ To our knowledge, only few cases with a prenatal diagnosis have been reported, even more seldom, are, reports with fetal autopsy correlations.

Uhl anomaly, arrhythmogenic right ventricular dysplasia (ARVD)/ cardiomyopathy, and right ventricular outflow tract tachycardia, are disorders that exhibit pathological changes involving the right ventricular myocardium. ${ }^{5}$ Except Uhl anomaly, the other two disorders are usually seen in late adolescence. Some have reported survival of cases of Uhl at 55 years of age and above. The severity of symptoms may vary and many patients may survive with their disease, which mainly depends on the presence of adequate number of intact myocardial cells. ${ }^{6}$ Detection of Uhl anomaly in early gestation allows prediction of the often poor chance of survival of the received

September 27, 2015

accepted

October 1, 2015

published online

November 2, 2015 fetus in utero in view of the possible development of severe cardiac dilatation, heart failure, pericardial effusion, and fetal hydrops.

Here, we report an antenatal case of Uhl anomaly, a rare finding in fetal echocardiography in a 33-year-old primigravida who presented at 20 weeks' of gestation. Cardiac ultrasound and further follow-up revealed severe RV dysfunction, cardiomegaly, and pericardial effusion as complications to Uhl anomaly. We were able to confirm the presence of this rare anomaly at the fetal autopsy, with histopathological examination.

\section{Case History}

A 33-year-old primigravida, who had conceived after an intracytoplasmic sperm insemination, was referred to the cardiac center at Parumala, at 20 weeks' gestational age (GA) for cardiac ultrasound evaluation. A routine ultrasound examination had shown a cardiac chamber enlargement. No
Copyright $\odot 2016$ by Thieme Medical Publishers, Inc., 333 Seventh Avenue, New York, NY 10001, USA. Tel: +1(212) 584-4662.
License terms

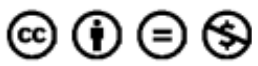

10.1055/s-0035-1566296. ISSN 2157-6998. 
significant medical or family history was contributory to any structural heart disease. The fetal heart rate was 180 beats per minute and fetal cardiac ultrasound examination showed an abnormally large $\mathrm{RV}$, in a routine four chamber view and even at the initial coronal sweep of the fetus ( $\mathbf{F i g}$. 1A, B). Cardiothoracic ratio showed significant cardiac dilatation, being $0.60,>95 \%$ percentile $(0.53$, 50th percentile at 20 weeks GA). The great arteries relationship was normal.

The RV was markedly dilated and the RV free wall was extremely thin at the basal portion with a paucity of apical trabeculations. RV dimension in width was $11 \mathrm{~mm},>95$ th percentile ( $7.8 \mathrm{~mm}$, 50th percentile at 20 weeks GA). The left ventricular width was $5.6 \mathrm{~mm},<5$ th percentile $(7.7 \mathrm{~mm}$, 50th percentile at 20 weeks GA). Fossa ovalis measured $3 \mathrm{~mm}$ and was not restricted. The anterior leaflet of the tricuspid valve was found to be abnormally bowing toward the small right auricle (RA) and the medial (septal) leaflet was abbreviated (-Fig. 1C). No tricuspid valve regurgitation was observed. There was a relative high gradient inflow at the tricuspid valve $(206 \mathrm{~cm} / \mathrm{s})$. The ventricular septum was intact. The mitral valve, pulmonary valve, and left ventricle were normal except for a mild decompression of the left ventricle due to RV enlargement (-Fig. 1C). Moderate posterior wall effusion was noticed during the second follow-up at the 23rd week of gestation. No other anomaly was observed on the scan performed at that time.

Differential diagnosis made at the time of the first visit, lay between an Ebstein spectrum and Uhl anomaly. A further ultrasound evaluation at the 23rd week of gestation, confirmed the diagnosis of Uhl anomaly. The parents were counselled regarding this rare anomaly, especially with regards to the survival of the fetus in utero, and its complications. The possible outcome and management after birth were clearly outlined, of a fetus with the rare anomaly of the heart with an obvious cardiomegaly, pericardial effusion, severe RV dysfunction, and heart failure.

Finally, the parents decided to have a termination of pregnancy. An autopsy was performed at 23rd week, where an abdominal solitus was seen. The liver was abnormally large and congested. Heart was in levocardia position, but, was huge, occupying almost the entire chest cavity and there was a moderate pericardial effusion. Inferior and superior venae cavae were draining normally into the right atrium. There was an aneurysmal dilatation of the RV. The lungs, atrial appendages, and great artery relationship were normal (-Fig. 2A-E).

The RV showed marked thinning of the lateral wall and anterior wall. The septal leaflet was attached to two abnormally elongated prominent conjoint septal papillary muscles in the inlet part of the ventricular septum. Anterior and posterior papillary muscles were found normally attached to both the anterior and posterior leaflets of the tricuspid valve with a paucity of RV apical trabeculations (-Fig. 3A). The RV anterior wall was abnormally thin and this was well demonstrated by a transillumination test confirming extensive loss of myocardium (-Fig. 3B). Pulmonary artery was normal with normal left and right pulmonary artery branches. The pulmonary cusps were normal (-Fig. 3C). The left ventricle, the ventricular septum, mitral valve, and left atrium, were normal. The rest of the right atrial wall was thickened ( - Fig. 3D-E). No other gross anomaly was noticed in the fetus.

Histopathological evaluation of specimens taken from the anterior wall of the RV stained with Masson trichrome, revealed fibrosis with lack of myocardium, (- Fig. 4A). Hematoxylin and eosin stain revealed well developed sinusoids around all fibers in the tissue, which appeared to consist of much thinned out myocardial cells or apparently poorly developed cardiac myocytes. The pericardium and endocardium appeared to enclose small groups of many cells some of which appeared to be developing cardio myocytes. There was marked interstitial edema with scant cardiac myocytes in one focal area. The rest of the fibers appeared to be in the development stages. The striking feature was the presence of screw-like wavy inclusions in the nuclei (-Fig. 4B) and this was seen in many cells.

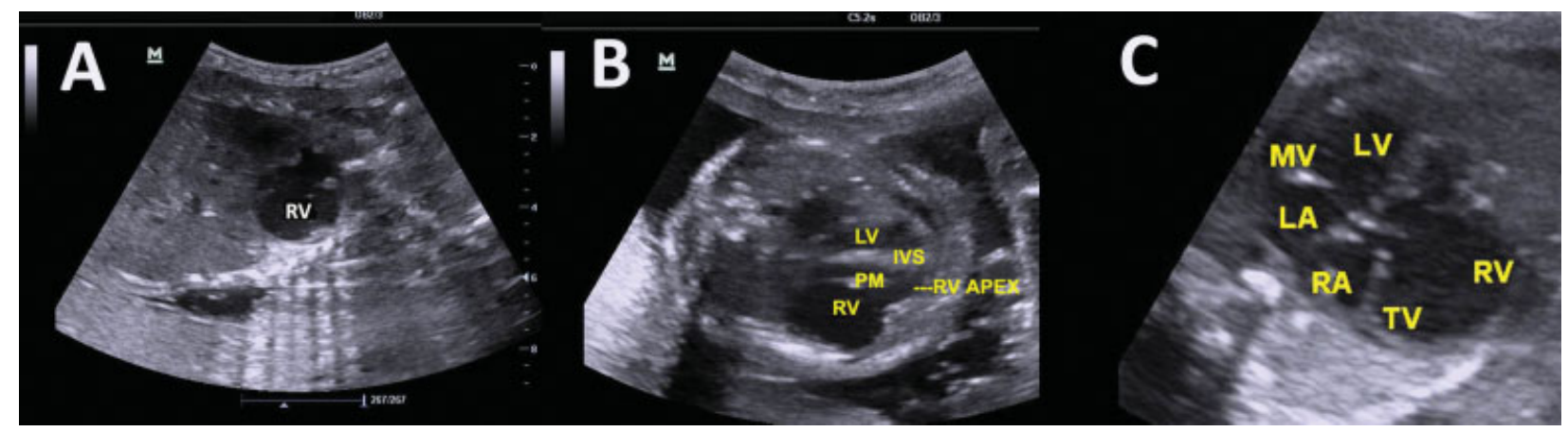

Fig. 1 (A-C) Fetal cardiac ultrasound images at 20 weeks GA. (A) Coronal sweep showed dilated large cardiac chamber. (B) Apical four chamber view showed, abnormally dilated heart occupying the entire chest cavity and strikingly enlarged upper portion of RV with preserved RV apex. (C) Normal left ventricle, interventricular septum, mitral valve, and dooming tricuspid septal and anterior cusp. GA, gestational age; IVS, interventricular septal thickness; LA, left auricle; LV, left ventricle; MV, mitral valve; PM, papillary muscle; RA, right auricle; RV, right ventricle; TV, tricuspid valve. 


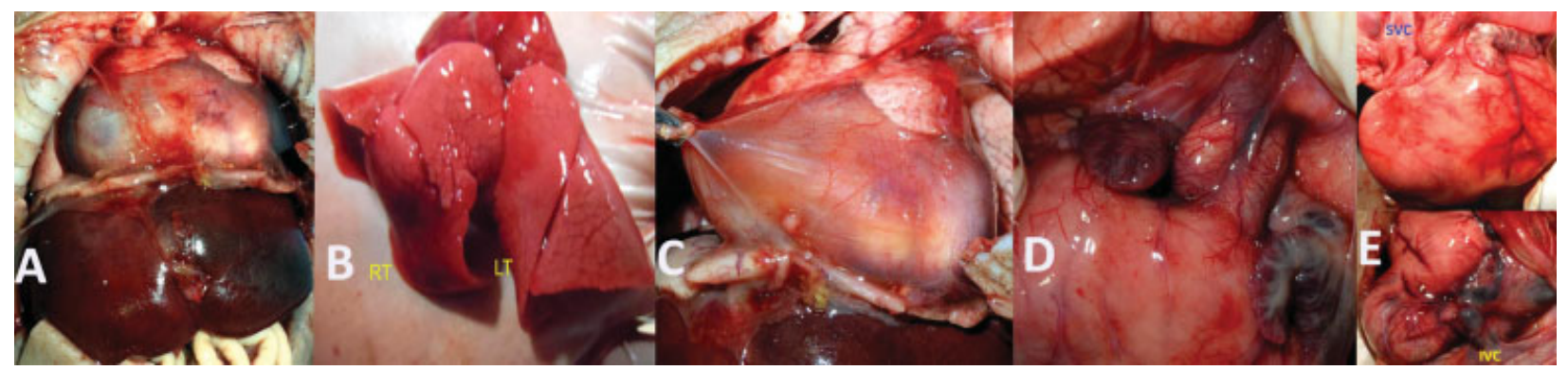

Fig. 2 (A-E) Autopsy images at 20 weeks GA. (A) Central incision open view showed huge congested liver extending to left hypochondrium with abnormally dilated heart occupying entire chest cavity. (B) Normally lobulated right and left lung. (C) Dilated heart with pericardial effusion in situ. (D) Normally related great vessels with appendages. (E) Normally related superior and inferior vena cava and dilated right ventricle identified between right and left coronaries. GA, gestational age; IVC, inferior vena cava; LT, left; RT, right; SVC, superior vena cava.

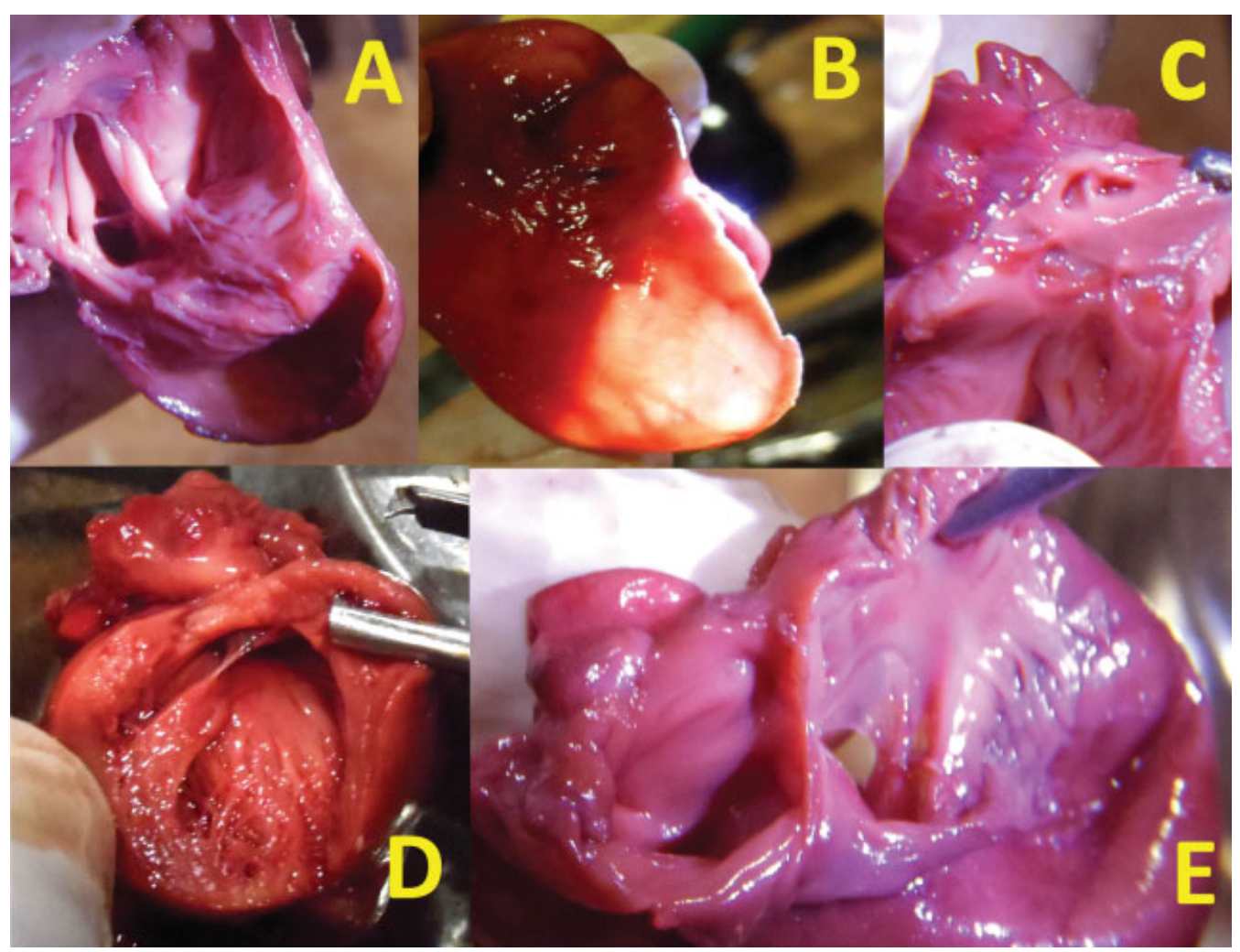

Fig. 3 (A-E) Autopsy images of heart. (A) Thin-walled RV free wall cut open view showed elongated conjoined septal papillary muscle with dooming septal tricuspid cusp. (B) Extremely thinned out RV free wall demonstrated at transillumination test. (C) Pulmonary artery cut open view shows normal tricuspid PV with orifices of LPA and RPA. (D) Normal ventricle with APM and PPM. (E) Atrium transverse cut open view shows dilated RA, prominent pectinate muscles. APM, anterior papillary muscle; LPA, left pulmonary artery; PPM, posterior papillary muscle; PV, pulmonary valve; RA, right auricle; RPA, right pulmonary artery; RV, right ventricle.

\section{Discussion}

Osler ${ }^{1}$ had first referred to a parchment heart in which all cardiac chambers were thinned out and dilated, though no clinical correlates were given. In 1952, Uhl described a case in which there was isolated RV dilatation, with thinned out free wall almost devoid of myocardium, an appearance akin to parchment heart. ${ }^{2}$ Since then, many case reports have been reported with either partial or total absence of myocardium in which the epicardium and endocardium apposed each other with normal tricuspid valve, intact ventricular septum and normal left ventricle. ${ }^{3,4,6}$ However, very few reports with a prenatal diagnosis and autopsy correlates have been published. The first reported prenatal case presented with thrombosis in RV. ${ }^{6}$

In the past, a diagnosis of Uhl anomaly was usually made only at autopsy. Currently, with the development of noninvasive imaging modalities, especially, early detection of the anomaly in intrauterine life can be accomplished with an accurate clinical diagnosis as demonstrated in the present case. In a study of more than 193 published cases, the ages at presentation, ranged from 1 day to 84 years; the presence of 


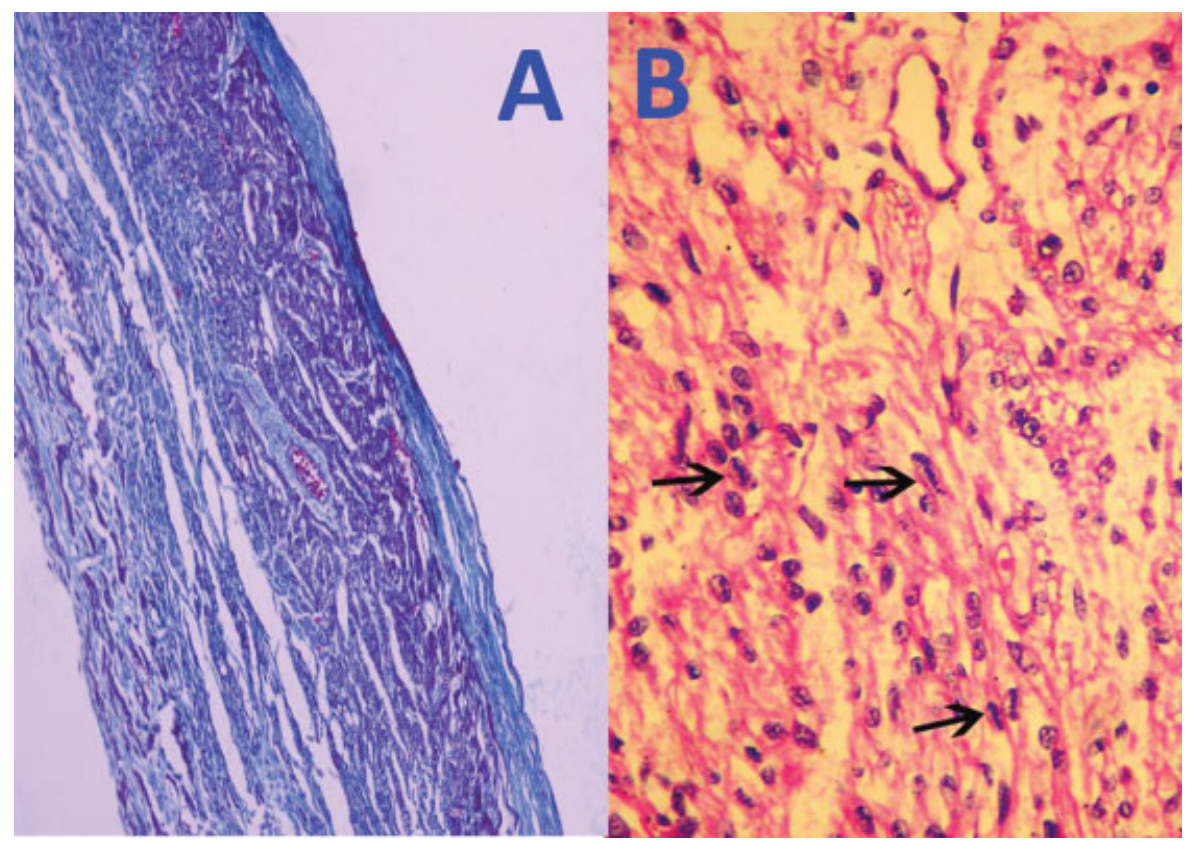

Fig. 4 (A) Masson trichrome stain from the anterior portion of RV myocardium showing fibrosis (blue). (B) Hematoxylin-eosin stain from the thinned out myocardium with scant myocytes. Arrows pointing to the abnormal wavy screw-like inclusions within the nuclei of the cells.

Uhl anomaly was detected in the young even in the early prenatal period, to late second trimester of gestation. ${ }^{3}$ Uhl anomaly is generally not associated with additional heart lesions as described in earlier studies. ${ }^{4}$ However, it could be associated with other associated congenital heart diseases. In a study of 481 cases, 17 cases were associated with cardiac malformations. ${ }^{7}$

Uhl anomaly should be distinguished from arrhythmogenic RV dysplasia (ARVD). Although, both appear to be separate entities with somewhat different morphology, further molecular genetic studies may unravel an overlap related to mutations in various forms of desmosome proteins. ARVD occurs at a later age being rarely seen before the age of 20. ${ }^{5,8}$ Today, we know that ARVD is an inherited genetic disease most frequently due to mutations in desmosome proteins, and pathologically there is replacement of myocardial cells by fibrofatty tissue.

The basic hemodynamic problem in Uhl disease, especially in fetal life is the deterioration of right ventricular function leading to insufficient RV contraction. Pulmonary and ductal flows are driven by paradoxical motion of interventricular septum and RA contraction $^{6}$ and right atrium eventually enlarges. Uhl had pointed out that the intractable nature of RV failure was due to the destruction of the crista supraventricularis and thereby loss of effective integration of left and right ventricular systolic function. Selective but unrestrained apoptosis of right ventricular myocytes after complete cardiac development has been postulated to explain Uhl anomaly. ${ }^{2,8}$ Bharati and $\mathrm{Lev}^{9}$ have reported 15 cases of Uhl anomaly and/or ARVD in their huge collection of over 6,300 congenital heart disease and while discussing the etiology, they have concluded that, "the susceptibility may lie in a specific type of abnormal formation of the myocardium itself at the molecular-genetic level during the early stages in the embryo genesis of the heart." Current trends in the discussion of etiology are indeed in favor of a genetic mutation with a varying intensity in desmosome proteins, resulting in the production of differing severity of Uhl anomaly and/or ARVD type of hearts. This might well be the case here, as we have demonstrated some nuclear abnormality in the present case, the striking feature being the abnormal wiry appearance within the nuclei of the cardiac myocytes which is probably related to genetic mutations.

Diagnosing Uhl anomaly in fetal life is essential since mortality and survival mainly depend on the severity of RV dysfunction related to, the either partial or complete absence of the myocardium. Hence, surviving cases need to be followed up carefully and counselled accordingly and multistage single ventricle correction or even orthotopic cardiac transplantation may be advised. ${ }^{10}$ The relationship, if any, of intracytoplasmic sperm insemination to Uhl anomaly should be further explored.

\section{Conflict of Interest}

None.

\section{Funding}

No external funding was received for this article.

\section{References}

1 Osler WM. The Principles and Practice of Medicine. 6th ed. New York, NY: D. Appleton; 1905:280

2 Uhl HSM. A previously undescribed congenital malformation of the heart: almost total absence of the myocardium of the right ventricle. Bull Johns Hopkins Hosp 1952;91(3):197-209 
3 Gerlis LM, Schmidt-Ott SC, Ho SY, Anderson RH. Dysplastic conditions of the right ventricular myocardium: Uhl's anomaly vs arrhythmogenic right ventricular dysplasia. Br Heart J 1993;69(2):142-150

4 Vecht RJ, Carmichael DJS, Gopal R, Philip G. Uhl's anomaly. Br Heart J 1979;41(6):676-682

5 Pamuru PR, Dokuparthi MV, Remersu S, Calambur N, Nallari P. Comparison of Uhl's anomaly, right ventricular outflow tract ventricular tachycardia (RVOT VT) \& arrhythmogenic right ventricular dysplasia/cardiomyopathy (ARVD/C) with an insight into genetics of ARVD/C. Indian J Med Res 2010;131(1):35-45

6 Cardaropoli D, Russo MG, Paladini D, et al . Prenatal echocardiography in a case of Uhl's anomaly. Ultrasound Obstet Gynecol 2006; 27(6):713-714
7 O' Connor WN, Cotterill CM, Marion MT, Noonan JA. Defective regional myocardial development and vasculirization in one variant of tricuspid atresia-clinical and necropsy findings in three cases. Cardiol Young 1992;2(1):42-52

8 Hébert JL, Duthoit G, Hidden-Lucet F, et al. Images in cardiovascular medicine. Fortuitous discovery of partial Uhl anomaly in a male adult. Circulation 2010;121(22):e426-e429

9 Bharati S, Lev M. The Pathology of Congenital Heart Disease. Armonk, NY: Futura Publishing Co; 1996:1524-1526

10 Ikari NM, Azeka E, Aiello VD, Atik E, Barbero-Marcial M, Ebaid M. Uhl's anomaly. Differential diagnosis and indication for cardiac transplantation in an infant. Arq Bras Cardiol 2001;77(1): 69-76 